\title{
An adaptive time-resolution method for ultra-short-term wind power prediction
}

\author{
Li, Lijuan; Li, Yuan; Zhou, Bin; Wu, Qiuwei; Shen, Xiaoyang; Liu, Hongliang; Gong, Zheng
}

Published in:

International Journal of Electrical Power and Energy Systems

Link to article, DOI:

10.1016/j.ijepes.2019.105814

Publication date:

2020

Document Version

Peer reviewed version

Link back to DTU Orbit

Citation (APA):

Li, L., Li, Y., Zhou, B., Wu, Q., Shen, X., Liu, H., \& Gong, Z. (2020). An adaptive time-resolution method for ultrashort-term wind power prediction. International Journal of Electrical Power and Energy Systems, 118, [105814]. https://doi.org/10.1016/j.ijepes.2019.105814

\section{General rights}

Copyright and moral rights for the publications made accessible in the public portal are retained by the authors and/or other copyright owners and it is a condition of accessing publications that users recognise and abide by the legal requirements associated with these rights.

- Users may download and print one copy of any publication from the public portal for the purpose of private study or research.

- You may not further distribute the material or use it for any profit-making activity or commercial gain

- You may freely distribute the URL identifying the publication in the public portal 


\title{
An adaptive time-resolution method for ultra-short-term wind power prediction
}

\author{
Lijuan $\mathrm{Li}^{\mathrm{a}}$, Yuan $\mathrm{Li}^{\mathrm{a}}$, Bin Zhou* ${ }^{\mathrm{b} *}$, Qiuwei $\mathrm{Wu}^{\mathrm{c}}$, Xiaoyang Shen ${ }^{\mathrm{d}}$, Hongliang $\mathrm{Liu}^{\mathrm{e}}$, Zheng Gong ${ }^{\mathrm{f}}$ \\ ${ }^{\mathrm{a}}$ College of Information Engineering, Xiangtan University, Xiangtan, 411105, China \\ ${ }^{\mathrm{b}}$ College of Electrical and Information Engineering, Hunan University, Changsha, 410082, China \\ ${ }^{\mathrm{c}}$ Department of Electrical Engineering, Technical University of Denmark, Lyngby, 2800, Denmark \\ ${ }^{\mathrm{d} S}$ chool of Foreign Languages, Xiangtan University, Xiangtan 411105, China \\ ${ }^{\mathrm{e}}$ College of Mathematics and Computational Science, Xiangtan University, Xiangtan 411105, China \\ ${ }_{\mathrm{f}}$ Zibo Power Supply Company, Shandong Electric Power Corporation, Zibo, 255000, China
}

\begin{abstract}
Accurate wind power prediction (WPP) plays an important role in the secure operation and dispatch of power systems. This paper proposes an adaptive time-resolution method to improve the accuracy of ultra-short-term wind power prediction (USTWPP). Firstly, the hidden prediction error (HPE) with its fluctuation magnitude and rate indicators is defined to reveal the fluctuation characteristics of real-time wind power. Then, the adjustment time of time-resolution can be dynamically determined by evaluating the fluctuation magnitudes, and the adjustment rules are formulated by mining the regularities of fluctuation rate of historical wind power data and establishing the interval grouping optimization model. Finally, by coupling the adjustment time and rules into the prediction model of back propagation neural network (BPNN), the rolling prediction with adaptive adjustment of time-resolution is achieved. Extensive tests have not only demonstrated the validity of the proposed method, but also confirmed its capability to cope with the USTWPP especially under situations of extremely violent fluctuations of wind power.
\end{abstract}

\section{Highlights}

An adaptive time-resolution method is proposed to improve the accuracy of USTWPP.

The HPE is defined to reveal the real-time fluctuation characteristics of wind power.

Fluctuation regularities are mined and grouped to formulate adaptive adjustment rules.

The proposed method can cope with the instantaneous USTWPP with violent fluctuations.

Keywords: Adaptive prediction, neural network, prediction error, prediction model, wind power prediction.

*E-mail addresses: binzhou@hnu.edu.cn (B. Zhou). 


\section{Introduction}

With the increasingly severe shortage of traditional fossil energy and environmental pollution problem, clean and renewable energy resources, especially wind energy, have been exploited and utilized on a large scale [1]. Accordingly, the penetration level of wind energy in the power systems is continuously rising. However, the random and intermittent nature of wind energy imposes challenges on the secure and stable operation of power systems [2], [3]. Therefore, it is of great significance to study the fluctuation characteristics of wind power and improve its prediction accuracy to promote the integration of wind power into power systems and improve the security and stability of the systems [4], [5].

WPP can be divided into long-term prediction, medium-term prediction, short-term prediction and ultra-short-term prediction according to the time scale. The USTWPP refers to rolling prediction of the wind power in the next few hours [6]. Improvement in the prediction accuracy of USTWPP can relieve the pressure of frequency regulation and optimize spinning reserve, which is beneficial to ensure the stability of the power systems as well as the reliable supply of power [7], [8].

The current prediction models of wind power mainly include two categories: physical models and statistical models [9]. The former involves complex calculation and the numerical weather prediction, a necessary step of the models, is time consuming [10], [11]. For these reasons, the physical models are not suitable for USTWPP, and thus not concerned in this paper. The statistical models are used to predict wind power by establishing the mapping relationship between input factors and output results based on the statistical historical data, such as time series [12], artificial neural network (ANN) [13], Kalman filter [14], grey prediction [15], support vector machine (SVM) [16], autoregressive integrated moving average (ARIMA) [17], extreme learning machine (ELM) [18], [19] and their improved models [20], [21]. The modelling principle of statistical models is simple and effective, and the prediction accuracy will decrease as the prediction horizon shortens. Therefore, the statistical models are suitable for USTWPP [10]. Among these models, ANN has the advantages of self-learning, self-organization, self-adaptation and a strong nonlinear function approximation ability so that it is the most widely used model in WPP [22]. Meanwhile, BPNN is one of the most popular ANN structures [23].

Recently, extensive research has been conducted to improve the prediction accuracy of ANN model for WPP. For instance, the wind data are processed by principal component analysis to select the key factors affecting wind speed fluctuation as the candidate inputs of BPNN, which can effectively reduce the prediction complexity [24]. The neural network model is combined with seasonal autoregressive integrated moving average to predict wind power based on data characteristics [25]. Gaussian process regression (GPR) is coupled into the internal structure of ANN for probabilistic wind speed prediction to main- 
1 tain the strong fitting ability of the deep learning methods and the probability characteristics of GPR [26]. Light gradient boosting machine algorithm is innovatively applied to the convolution neural network model to predict ultra-short-term wind power, which overcomes the limitations of the single-convolution model in WPP [27]. In the above models, the wind speed or wind power is both predicted with fixed time-resolution, that is, the input and output data and the rolling prediction of the prediction model are periodic with a fixed time step. For instance, $15 \mathrm{~min}$ is taken as the time-resolution of USTWPP in [11] and $[25]$.

However, the historical measured data of large-scale wind farms show that the minute-level fluctuations of wind power sometimes have the characteristics of large magnitude and rate of change. The largest 10-min wind power fluctuation of North Bridge Wind Farm in Jiuquan, China in 2009 accounted for 35\% of the installed wind capacity [28]. Moreover, in Texas power system, there was a 10-min wind power fluctuation of $1256 \mathrm{MW}$, which accounted for $14 \%$ of the installed wind capacity in 2009 . And the emergency events on February 24, 2007 and February 26, 2008 were caused by the large minute-level fluctuations of wind power in Texas power system [29]. It can be seen that the USTWPP model with fixed time-resolution (usually $15 \mathrm{~min}$ ) cannot track the large minute-level fluctuations of wind power accurately, which will result in large instantaneous prediction error within the time step. The instantaneous prediction error will bring great uncertainty to the real-time dispatching of power systems with 5-min time period [30], [31], so as to seriously threaten the security and stability of power systems and reduce the ability of the system to integrate wind power [32].

The time-resolution of WPP model contradictorily affects the prediction accuracy and prediction efficiency. Therefore, an adaptive time-resolution method for USTWPP is proposed in this paper to reduce the instantaneous prediction error and improve the prediction accuracy to the benefit of safe and stable operation of power system.

The main contributions of this paper can be listed as follows:

1) The HPE is defined and evaluated for real-time USTWPP to reveal instantaneous dynamic characteristics of wind power prediction error within a rolling time step, and it can overcome the shortcomings of the existing prediction error indicators only representing the average prediction performance within the time step.

2) An adaptive time-resolution method is proposed for USTWPP to dynamically identify the lookahead prediction time-resolution along the timeline based on the fluctuation characteristics of HPE. With the dynamic adjustment of time-resolution, higher prediction accuracy is achieved especially under the situations of extremely violent fluctuations of wind power. 
The rest of the paper is organized as follows: The definition and characteristic analysis of HPE are described in Section 2. An adaptive time-resolution method, including adjustment time and rules of time-resolution, is introduced in Section 3. Comparative studies between the performance of the proposed prediction model and that of benchmark prediction models are provided in Section 4. The paper is concluded in Section 5.

\section{Definition and characteristic analysis of hidden prediction error}

A large degree of instantaneous prediction error could occur in USTWPP with fixed time-resolution because of the violent minute-level fluctuations of wind power. Obviously, an effective dispatch schedule in power systems with wind power depends on the credible and precise USTWPP with a less degree of prediction error. Understanding the characteristics of prediction error is the basis of improving the prediction accuracy of WPP. Therefore, it is important to describe prediction error so that effective measures could be taken to decide on a proper time-resolution in USTWPP. Here, HPE is defined and the evaluation indicators of fluctuation magnitude and fluctuation rate are established to describe the instantaneous prediction error within the time step and reveal real-time fluctuation characteristics.

\subsection{Definition of hidden prediction error}

Generally, wind power is predicted with fixed time-resolution. For instance, 'Function Specification of Wind Power Prediction' in China's power industry standards stipulates that the time-resolution of USTWPP $T_{u s}$ is not less than $15 \mathrm{~min}\left(T_{u s} \leq 15 \mathrm{~min}\right)$ [33]. Correspondingly, the evaluation indicators of prediction error are calculated based on the prediction with fixed time-resolution. As shown in (1) and (2), the prevailing wind power prediction error is a description of average prediction performance within a time step (hereinafter referred to as average prediction error). The absolute average prediction error $e_{a b s}$ represents the difference between the average actual values $P_{M}$ and the average predicted values $P_{P}$ [34]. The relative average prediction error $e_{r e l}$ is the ratio of $e_{a b s}$ to the total capacity of the wind farm $S_{o p}$.

$$
\begin{gathered}
e_{a b s}=P_{M}-P_{P} \\
e_{r e l}=\frac{P_{M}-P_{P}}{S_{o p}}
\end{gathered}
$$

The $e_{a b s}$ and $e_{r e l}$ can only represent the average values of wind power prediction error within every time step, but cannot reflect the dynamic fluctuations of prediction error within the time steps. That is, the instantaneous prediction errors are undetectable. Especially, when wind power has the characteris- 
tics of violent minute-level fluctuations, large instantaneous prediction errors are ignored, which brings great uncertainty to the real-time dispatching of power systems and then threatens the security and stability of the power systems. Therefore, for USTWPP, besides the average prediction error, the instantaneous prediction error within the time step should be concerned as well. By accurately describing the real-time fluctuation characteristics of wind power and the tracking effect of prediction model for the real-time fluctuations, the instantaneous prediction error will provide important insights for the improvement of the prediction accuracy. Because the instantaneous prediction error is hidden within the time step, HPE is defined in this paper.

According to the regulations of data collection and processing in 'Function Specification of Wind Power Prediction' [33], the sampling period of real-time power in a wind farm should be no longer than 1 min; the system stores power data during the whole operation process and converts the stored data into 15-min average data for fixed time-resolution prediction and error analysis, which are referred to as average actual recorded values. Therefore, in this paper, $1 \mathrm{~min}$ is taken as the sampling period of real-time wind power and is selected as the calculation time period of HPE $T_{H E}\left(T_{H E}=1 \mathrm{~min}\right)$ to analyse the instantaneous prediction error. Hence, the absolute HPE $e_{\text {Habs }}$ and relative HPE $e_{\text {Hrel }}$ of wind power are given as:

$$
\begin{gathered}
e_{\text {Habs }}=P_{M H}-P_{P H} \\
e_{H r e l}=\frac{P_{M H}-P_{P H}}{S_{o p}}
\end{gathered}
$$

where $P_{M H}$ is the set of actual measured values of wind power with 1-min sampling period; $P_{P H}$ is the set of equivalent predicted values of wind power with 1-min period linearly converted from the power values predicted with time-resolution of $T_{u s}$.

Here, the wind power prediction error in Fig. 1 is taken as an example for analysis. The $e_{\text {rel }}$ and maximum value of $e_{\text {Hrel }}$ within each time step are shown in Table 1. Moreover, the relative deviation between $e_{\text {rel }}$ and the maximum value of $e_{\text {Hrel }}$ is listed in the fourth column of the table, which is calculated by dividing the difference between the maximum value of $e_{H r e l}$ and $e_{\text {rel }}$ by $e_{\text {rel }}$. It can be seen that the maximum value of $e_{\text {Hrel }}$ is larger than the corresponding $e_{\text {rel }}$ within each time step. Especially, the relative deviation within the time step $T_{1} \sim T_{2}$ reaches up to $43.35 \%$. Due to the existence of a large relative deviation, HPE deserves our attention. The large HPE will cause a severe power imbalance in the power system dispatching and thus cannot ensure secure operation of the systems. Therefore, it is necessary to evaluate and reduce HPE to improve the prediction accuracy of wind power. 


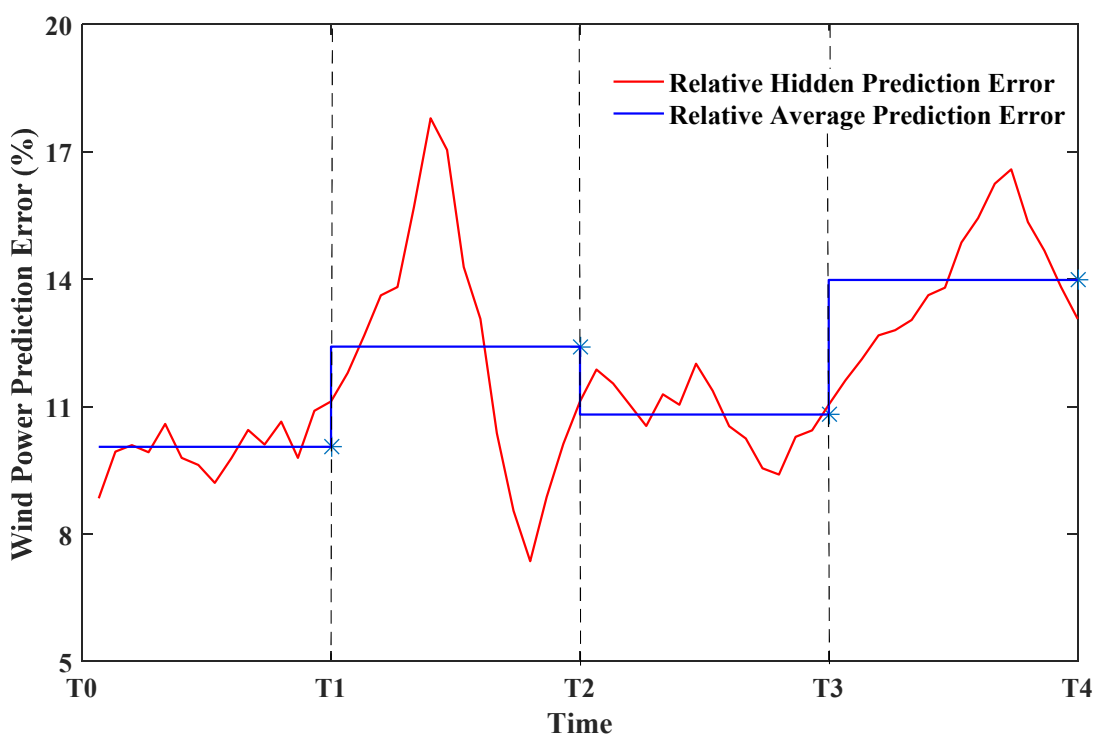

Fig. 1 Wind power prediction error

Table 1 Comparison between $e_{\text {rel }}$ and the maximum value of $e_{H r e l}$

\begin{tabular}{cccc}
\hline Time step & $e_{\text {rel }}(\%)$ & Maximum value of $e_{\text {Hrel }}(\%)$ & Relative deviation (\%) \\
\hline$T_{0} \sim T_{1}$ & 10.06 & 11.12 & 10.54 \\
$T_{1} \sim T_{2}$ & $\mathbf{1 2 . 4 1}$ & $\mathbf{1 7 . 7 9}$ & $\mathbf{4 3 . 3 5}$ \\
$T_{2} \sim T_{3}$ & 10.82 & 12.01 & 11.00 \\
$T_{3} \sim T_{4}$ & 13.98 & 16.59 & 18.97 \\
\hline
\end{tabular}

\subsection{Evaluation indicators of hidden prediction error}

At present, the existing evaluation indicators of WPP are shown in Table 2. These indicators are all utilized to evaluate the characteristics of the average prediction error within a time interval consisting of multiple time steps. Among them, mean absolute error (MAE) and root mean square error (RMSE) are used to measure the average magnitude and the dispersion of average prediction error within a time interval respectively [35]. The indicators $\delta_{a b s}$ and $\delta_{\max }$ are the maximum prediction error and maximum error rate, indicating the maximum risk resulted from the prediction error, which are a basis for establishing evaluation indicators of HPE. Accuracy $(A)$ will be adopted to evaluate the prediction results in case studies and thus it is also listed in the table. 
Table 2 Existing evaluation indicators of average prediction error

\begin{tabular}{cc}
\hline Evaluation indicator & Calculation formula \\
\hline mean absolute error $(M A E)$ & $M A E=\sum_{i=1}^{n}\left(\frac{\left|P_{M i}-P_{P i}\right|}{S_{o p} \times n}\right)$ \\
root mean square error $(R M S E)$ & $R M S E=\frac{\sqrt{\sum_{i=1}^{n}\left(P_{M i}-P_{P i}\right)^{2}}}{S_{o p} \times \sqrt{n}}$ \\
maximum prediction error $\left(\delta_{a b s}\right)$ & $\delta_{a b s}=\max \left(\left|P_{M i}-P_{P i}\right|\right)$ \\
maximum error rate $\left(\delta_{\max }\right)$ & $\delta_{\max }=\max \left(\frac{\left|P_{M i}-P_{P i}\right|}{S_{o p}}\right)$ \\
accuracy $(A)$ & $A=\left(1-\sqrt{\frac{1}{n} \sum_{i=1}^{n}\left(\frac{P_{M i}-P_{P i}}{S_{o p}}\right)^{2}}\right)$ \\
\hline
\end{tabular}

(Note: In the table, $n$ is the total number of samples with fixed time-resolution within a time interval; $P_{M i}$ and $P_{P i}$ are the $i$ th average actual recorded sample and the $i$ th average predicted sample of wind power, respectively.)

Accurate characteristic analysis of wind power prediction error is one of the most significant prerequisites for improving prediction accuracy. Therefore, besides the characteristic evaluation of average prediction error by the indicators in Table 2, the fluctuation characteristics of HPE within the time step should be analysed and evaluated, as the basis for adaptively adjusting the time-resolution of prediction model. Analysis of the fluctuation characteristics of HPE specifically includes the following aspects:

1) Fluctuation magnitude analysis: in order to detect whether there are wind power fluctuations with a large magnitude ignored in the prediction with time-resolution of $T_{u s}$, the fluctuation magnitude of HPE within a given time interval should be analysed.

2) Fluctuation rate analysis: in order to detect whether there are wind power fluctuations with a large rate of change and to measure the tracking effect of prediction model for the real-time fluctuations, the fluctuation rate of HPE within a given time interval should be evaluated.

To measure the maximum value of HPE and then detect the wind power fluctuation with maximum risk, the maximum hidden prediction error $\delta_{a b s H}$ and the maximum hidden error rate $\delta_{\max H}$ are established as the indicators for evaluating the fluctuation magnitude of HPE, as shown in (5) and (6), respectively.

$$
\begin{aligned}
\delta_{a b s H} & =\max \left(\left|P_{M H}-P_{P H}\right|\right) \\
\delta_{\max H} & =\max \left(\frac{\left|P_{M H}-P_{P H}\right|}{S_{o p}}\right)
\end{aligned}
$$


The violent minute-level fluctuations of wind power and wind power ramp behaviour both have the characteristics of a high magnitude and short duration [29]. Therefore, to indicate the fluctuation rate of HPE and the tracking effect of prediction model for the real-time fluctuations, the maximum average monotonic fluctuation rate $\rho_{\max H} \%$ is established as the fluctuation rate evaluation indicator of HPE based on the characteristics of ramp behaviour, as shown in (7).

$$
\rho_{\max H} \%=\max \left(\frac{\Delta e_{H c d}}{\Delta t_{H c d} \times S_{o p}} \times 100 \%\right)
$$

where the subscripts $c$ and $d$ represent the starting and ending points of a monotonic fluctuation (monotonic increase or monotonic decrease) of HPE within a time interval, respectively; $\Delta e_{H c d}$ and $\Delta t_{H c d}$ are the magnitude and duration of the monotonic fluctuation with the starting point $c$ and the ending point $d$, respectively. According to the statistical analysis of ramp behaviour characteristics in [29], let $\Delta t_{H c d}$ be greater than 2 min to avoid the interference of small fluctuations with an extremely short duration to the evaluation of $\rho_{\max H} \%$ and hence to improve the accuracy of fluctuation characteristic analysis. Meanwhile, other indicators are also established to evaluate HPE, including mean absolute hidden error $M A E_{H}$, root mean square hidden error $R M S E_{H}$ and hidden accuracy $A_{H}$, as shown in (8) $\sim(10)$.

(1)

where $Z$ is the total number of samples with the calculation time period of HPE $T_{H E}$ within a time interval; $P_{M H j}$ and $P_{P H j}$ are the $j$ th actual measured sample and the $j$ th equivalent predicted sample of wind power, respectively.

\subsection{Threshold setting of hidden prediction error evaluation indicator}

The evaluation criteria for wind power prediction error are developed according to the development of wind power and performance requirements of the power systems in different countries. For instance, 'Prediction and Management of Wind Farm Power' issued by the National Energy Administration of 
1 China stipulates that the real-time prediction error of wind power prediction system does not exceed $15 \%$ [34]. Therefore, the maximum hidden error rate threshold for USTWPP $\delta_{\text {usth }}$ is set as 0.15 .

\section{Time-resolution adaptive adjustment method}

In the WPP model, time-resolution directly affects the prediction accuracy and prediction efficiency. Larger time-resolution corresponds to higher prediction accuracy, a longer calculation time and a larger storage space for the prediction process. On the contrary, tinier time-resolution corresponds to lower prediction accuracy, a shorter calculation time and a smaller storage space. Therefore, it is necessary to adaptively adjust the time-resolution to make the prediction model track the real-time wind power fluctuations more accurately and to ensure the prediction efficiency in USTWPP.

\subsection{Adjustment time of time-resolution}

From the above analysis, it is obvious that there is large HPE in USTWPP with fixed time-resolution when the minute-level fluctuations of wind power are violent. Once the magnitude of HPE is large, it will threaten the secure and stable operation of the power systems. Therefore, the time-resolution should be adjusted to decrease HPE until HPE meets the evaluation criteria for USTWPP.

As there is a great temporal correlation in ultra-short-term wind power series [27], the corresponding HPE series calculated from wind power also has a great temporal correlation. Therefore, the fluctuation trend of HPE within the prediction target time interval (e.g., a prediction target time interval of USTWPP in the Chinese wind power prediction system is four hours) can be predicted based on the characteristics of HPE within the past adjacent time steps. The HPE within $N$ time steps before the starting point of prediction target time interval can provide a basis for the adaptive adjustment of time-resolution, which is taken as the reference data for fluctuation characteristic analysis.

The fixed time-resolution of prediction model is set as the initial reference time-resolution $T_{\text {us } 0}$ and the starting point of prediction target time interval is denoted by $t_{0}$. The steps for determining when to adjust the time-resolution are listed below.

Step 1: The wind power data within $N$ time steps before time $t_{0}$ are extracted, including the average predicted values $P_{P}$ with time-resolution $T_{u s 0}$ and 1-min actual measured values $P_{M H}$ (i.e., $N$ average predicted values and $T_{u s 0} \times N$ actual measured values).

Step 2: According to (4), the relative HPE $e_{\text {Hrel }}$ within the $N$ time steps are calculated.

Step 3: According to $(5) \sim(7)$, the maximum hidden error rate $\delta_{\max H}$ and the maximum average 
1 monotonic fluctuation rate $\rho_{\max H} \%$ of $e_{\text {Hrel }}$ within the $N$ time steps are calculated. The initial unadjusted $\rho_{\max H} \%$ of prediction with reference time-resolution $T_{u s 0}$ is denoted as $\rho_{\max H 0} \%$.

Step 4: The maximum hidden error rate $\delta_{\max H}$ is compared with the threshold $\delta_{\text {usth }}$. There are two scenarios about the comparison result.

Scenario 1: The calculated maximum hidden error rate $\delta_{\max H}$ is less than or equal to the threshold $\delta_{\text {usth }}\left(\delta_{\max H} \leq \delta_{\text {usth }}\right)$, which means that there is no wind power fluctuation with a large magnitude within the $N$ time steps. For this case, there is no need to adjust the time-resolution and consequently, the prediction model still adopts reference time-resolution $T_{u s 0}$ to predict the wind power within the prediction target time interval.

Scenario 2: The calculated maximum hidden error rate $\delta_{\max H}$ is greater than the threshold $\delta_{\text {usth }}$ $\left(\delta_{\max H}>\delta_{u s t h}\right.$ ), which indicates that there is wind power fluctuation with a large magnitude within the $N$ time steps. Thus, there will be large HPE in the USTWPP with reference time-resolution $T_{u s 0}$ and it is necessary to adjust the time-resolution within the prediction target time interval.

\subsection{Adjustment rules of time-resolution}

After determining the adjustment time of time-resolution, effective adjustment rules should be formulated to ensure that the prediction model can not only make the maximum hidden error rate $\delta_{\max H}$ meet the performance requirement, but also have high prediction efficiency.

In the adaptive adjustment of time-resolution, an adjustment coefficient $k_{u s}$ is adopted, and then the reference time-resolution $T_{u s 0}$ is adjusted to $T_{u s}^{\prime}$ :

$$
T_{u s}^{\prime}=k_{u s} \times T_{u s 0}
$$

where $T_{u s}^{\prime}$ is the new time-resolution of USTWPP after adjustment. The larger the initial maximum average monotonic fluctuation rate $\rho_{\max H 0} \%$ (i.e., the wind power fluctuation is more violent), the larger the time-resolution and the smaller the adjustment coefficient $k_{u s}$. Hence, the adjustment coefficient $k_{u s}$ is determined by the initial maximum average monotonic fluctuation rate $\rho_{\max H 0} \%$. The proper adjustment coefficient $k_{u s}$ will make the prediction model meet the prediction accuracy requirements and ensure the prediction efficiency.

Therefore, in order to obtain the proper adjustment coefficients $k_{u s}$, the adjustment rules are formulated by performing the following two processes. The regularities are mined from historical wind power data first. Then the regularities are grouped to formulate adjustment rules.

(1) Regularities mining 
The regularities of the relationship between $\rho_{\max H 0} \%$ and $k_{u s}$ are mined from historical wind power data according to the following four steps.

Step 1: Establishment of the mining data set

The mining data set for USTWPP from the historical wind power data is established, including the actually measured values with 1-min sampling period $P_{M H}$ and the average predicted values with 15-min fixed time-resolution $P_{P}$ within each prediction time interval of USTWPP.

Step 2: Fluctuation characteristics analysis of HPE

According to $(4) \sim(7)$, the initial relative HPE $e_{\text {Hrel }}$ with the fixed time-resolution and the corresponding maximum hidden error rate $\delta_{\max H}$ and maximum average monotonic fluctuation rate $\rho_{\max H 0} \%$ within the prediction time interval are calculated.

Step 3: Iterative adjustment of time-resolution

The calculated maximum hidden error rate $\delta_{\max H}$ is compared with the threshold $\delta_{u s t h}$. When $\delta_{\max H} \leq \delta_{u s t h}$, adjustment coefficient $k_{u s}$ is set as 1 so as to keep the time-resolution unchanged; otherwise an iterative adjustment is carried out until $\delta_{\max H}$ meets the prediction performance requirements.

During the iteration adjustment process, the time-resolution is decreased by 1 min every time. The step of adjustment coefficient refers to the change size of the adjustment coefficient to decrease time-resolution by $1 \mathrm{~min}$ every time. Therefore, the step of adjustment coefficient can be formulated as follows:

$$
l=\frac{1 \mathrm{~min}}{T_{\text {us } 0}}
$$

where $l$ is the step of adjustment coefficient $k_{\text {us }}$. For example, $l$ is $1 / 15$ when the reference fixed time-resolution $T_{u s 0}$ is $15 \mathrm{~min}$.

As long as the maximum hidden error rate $\delta_{\max H}$ is greater than the threshold $\delta_{\text {usth }}$ in the iteration, the adjustment coefficient $k_{u s}$ is changed as follows.

$$
k_{\text {us }}=1-h \times l \quad h=1,2,3, \cdots, H
$$

where $h$ is the number of times changing the adjustment coefficient; $H$ is the total number of times changing the adjustment coefficient in the whole iteration process when the maximum hidden error rate $\delta_{\max H}$ is changed from greater than the threshold $\delta_{\text {usth }}$ to less than or equal to the threshold $\delta_{\text {usth }}$.

Step 4: Determining the relationship between $\rho_{\max H 0} \%$ and $k_{u s}$

For any initial maximum average monotonic fluctuation rate $\rho_{\max H 0} \%$, the obtained $k_{u s}$ at the end of the iteration is taken as the corresponding adjustment coefficient. Consequently, one regularity of the relationship between $\rho_{\max H 0} \%$ and $k_{u s}$ is obtained. 
When the whole mining of the entire data set is completed, the regularities of the relationship between $\rho_{\max H 0} \%$ and $k_{u s}$ are summarized. Otherwise, the next mining cycle is executed.

(2) Grouping optimization of regularities

The fluctuation characteristics of wind power change frequently. If the regularities of the relationship between $\rho_{\max H 0} \%$ and $k_{u s}$ are directly applied to adjust $T_{u s 0}$, the adjustments will occur frequently and thus the prediction efficiency will be reduced. Therefore, it is necessary to divide $\rho_{\max H 0} \%$ and $k_{u s}$ into multiple groups and select a unique value from each group of $k_{u s}$ as the common adjustment coefficient of the corresponding group of $\rho_{\max H 0} \%$. The steps of interval grouping of $\rho_{\max H 0} \%$ and $k_{u s}$ are as follows:

Step 1: Determining the number of groups $M$

Considering the requirements of prediction accuracy and prediction efficiency, the number of groups $M$ is determined according to the length of series $k_{u s}$.

Step 2: Interval grouping optimization model

Here, an interval grouping optimization model is built. In order to ensure the adjustment effectiveness of each selected adjustment coefficient, series $k_{\text {us }}$ are grouped as evenly as possible. Therefore, the objective function of interval grouping optimization is to minimize the variance of group intervals of all adjustment coefficient groups, as shown in (14).

$$
\min \frac{1}{M} \sum_{m=1}^{M}\left[\left(k_{u s m}^{+}-k_{u s m}^{-}\right)-\mu\left(k_{u s m}^{+}-k_{u s m}^{-}\right)\right]^{2}
$$

where $k_{u s m}^{-}$and $k_{u s m}^{+}$are the lower and upper limits of the $m$ th adjustment coefficient group, respectively, $m \in(1, M) ; \mu(\bullet)$ represents the mean of $(\bullet)$ :

$$
\mu\left(k_{u s m}^{+}-k_{u s m}^{-}\right)=\frac{k_{u s \max }-k_{u s \min }}{M}
$$

where $k_{\text {usmin }}$ and $k_{\text {usmax }}$ are the minimum and maximum values of $k_{\text {us }}$, respectively.

The following constraints are considered. Because of the exhaustion principle of statistical grouping, the upper limit $k_{u s M}^{+}$and lower limit $k_{u s 1}^{-}$satisfy:

$$
\begin{gathered}
k_{u s 1}^{-}=k_{u s \min } \\
k_{u s M}^{+}=k_{u s \max }
\end{gathered}
$$

Due to the mutual exclusion principle, interval grouping needs to follow (17).

$$
k_{u s m}^{+}=k_{u s(m+1)}^{-} \quad \forall m \in[1, M)
$$

Considering that the real-time sampling period of wind power and the dispatching time period of power grid are both integer multiples of $1 \mathrm{~min}$ (i.e., $1 \mathrm{~min}$ and $5 \mathrm{~min}$, respectively), all the 
1 time-resolutions $T_{u s}^{\prime}$ adjusted by the adjustment coefficient $k_{u s}$ should be integers. Therefore, the constraints (18) and (19) should be satisfied.

$$
\begin{array}{ll}
k_{\text {usm }}^{+} \times T_{\text {us } 0} \in N_{+} & \forall m \in[1, M) \\
k_{\text {usm }}^{-} \times T_{\text {us } 0} \in N_{+} & \forall m \in(1, M]
\end{array}
$$

The power grid dispatching, one of the main applications of wind power prediction data, takes $5 \mathrm{~min}$ as the time period. To improve the convenience of wind power prediction data application in power grid dispatching, the key adjustment coefficients $k_{\text {us }}$ corresponding to time-resolutions of $5 \mathrm{~min}$ and $10 \mathrm{~min}$ should be retained to be the group limits. Hence, the constraints about the retention of key coefficients are shown in (20) and (21).

$$
\begin{aligned}
& \prod_{m=1}^{M}\left(k_{u s m}^{-} \times T_{u s 0}-5\right) \times\left(k_{u s m}^{+} \times T_{u s 0}-5\right)=0 \\
& \prod_{m=1}^{M}\left(k_{u s m}^{-} \times T_{u s 0}-10\right) \times\left(k_{u s m}^{+} \times T_{u s 0}-10\right)=0
\end{aligned}
$$

Based on (14) (21), the interval grouping optimization model is formed. The values of $k_{u s m}^{-}$and $k_{u s m}^{+}$are obtained by solving the model with cutting plane method.

Step 3: Obtaining the groups of $\rho_{\max H 0} \%$

Based on the relationship between $\rho_{\max H 0} \%$ and $k_{u s}, \rho_{\max H 0} \%$ corresponding to $k_{u s m}^{-}$and $k_{u s m}^{+}$ can be found, and then the groups of $\rho_{\max H_{0}} \%$ can be obtained, as shown in (22).

$$
\left(\rho_{\max 0} \%_{m}^{-}, \quad \rho_{\max H 0} \%_{m}^{+}\right] \quad m \in[1, M]
$$

where the lower limit $\rho_{\max H_{0}} \%_{m}^{-}$and upper limit $\rho_{\max { }_{0}} \%_{m}^{+}$are $\rho_{\max H 0} \%$ corresponding to $k_{\text {usm }}^{+}$and $k_{\text {usm }}^{-}$, respectively.

Step 4: Selecting the common adjustment coefficient $k_{u s m}$ corresponding to each group of $\rho_{\max H 0} \%$

Since the lower limit $k_{u s m}^{-}$corresponds to the most violent wind power fluctuation in each adjustment coefficient group, the common adjustment coefficient $k_{u s m}$ corresponding to each group of $\rho_{\max H 0} \%$ is set as $k_{u s m}^{-}\left(k_{u s m}=k_{u s m}^{-}\right)$so as to meet the prediction accuracy requirement.

In conclusion, the flowchart of establishing the adjustment rules of the time-resolution of USTWPP is illustrated in Fig. 2.

\subsection{Rolling prediction with adaptive adjustment of time-resolution}

By coupling the time-resolution adjustment method including adjustment time and adjustment rules into the current prediction model, such as popular BPNN, the rolling prediction with adaptive adjustment of time-resolution is conducted as follows: 


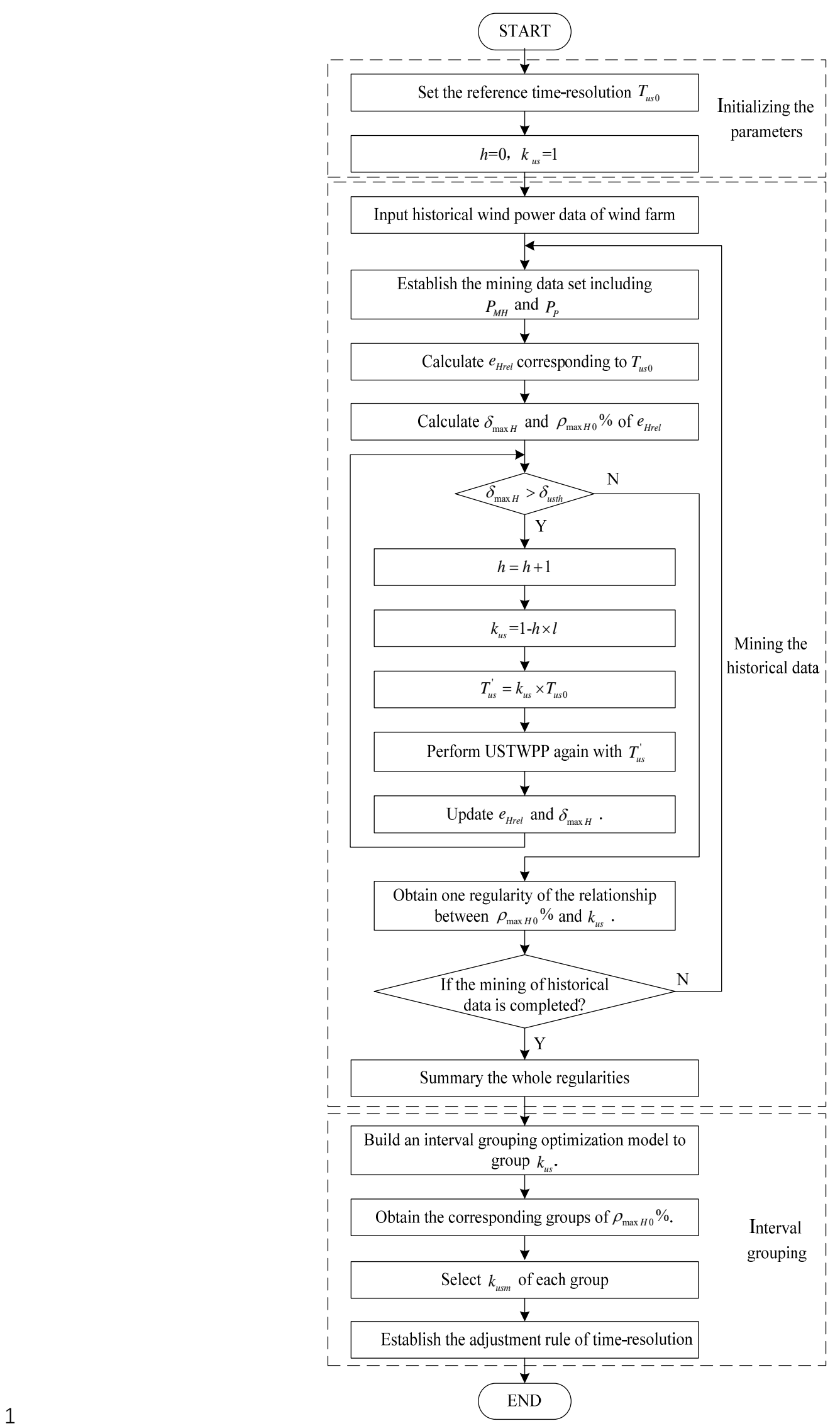

Fig. 2 Flowchart of establishing the adjustment rules of time-resolution of USTWPP 
Step 1: The prediction target time interval and its starting point $t_{0}$ are set. flowchart is given in Fig. 3.

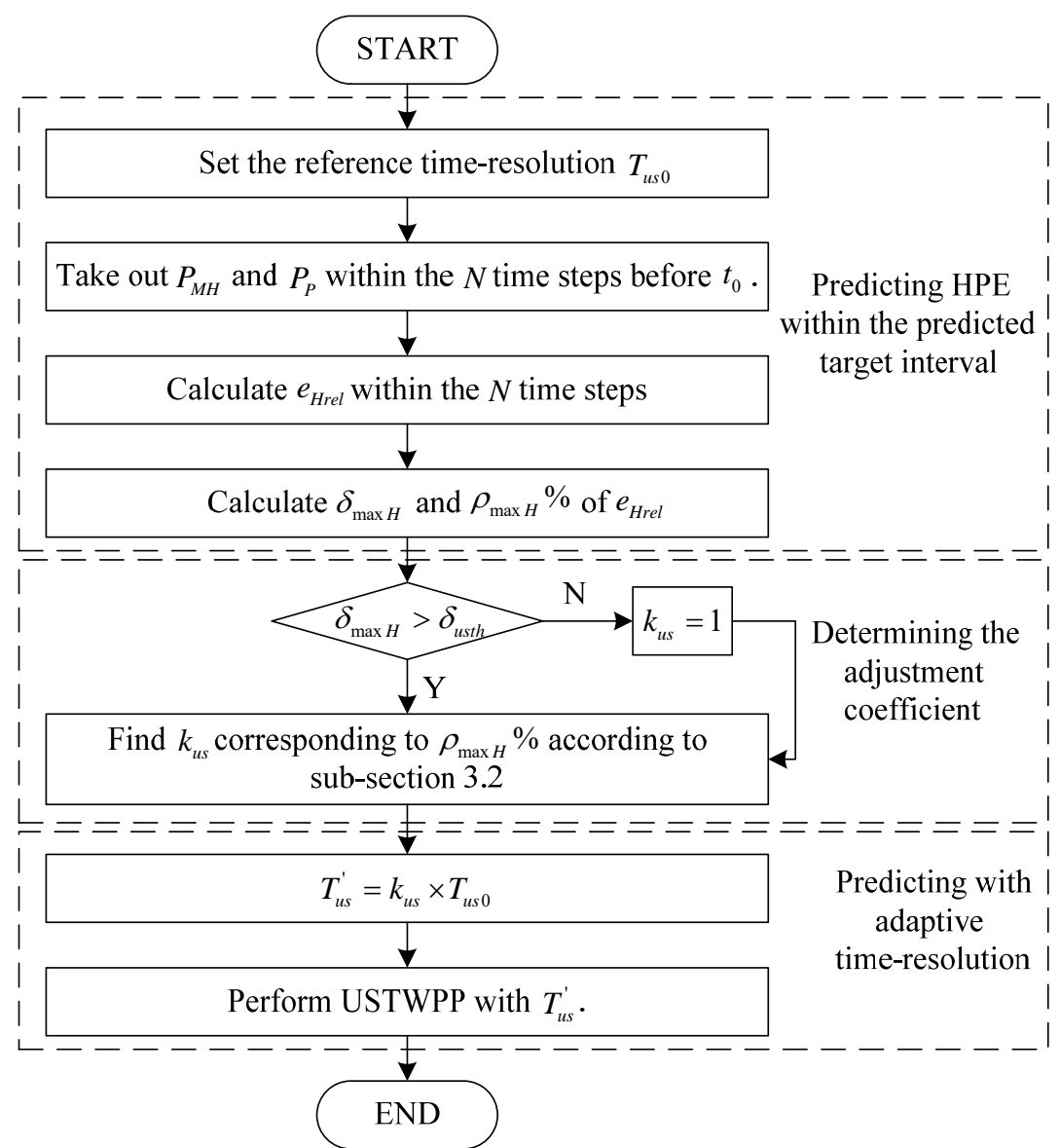

Fig. 3 Flowchart of prediction with adaptive time-resolution

Firstly, the fluctuation characteristics of HPE (i.e., $\delta_{\max H}$ and $\rho_{\max H} \%$ ) with reference fixed time-resolution $T_{\text {us } 0}$ within the prediction target time interval are predicted, and whether or not to adjust the time-resolution is judged according to sub-section 3.1.

Then, based on the adjustment rules in the sub-section 3.2, the corresponding adjustment coefficient of time-resolution $k_{u s}$ is found.

Finally, the time-resolution $T_{u s}^{\prime}$ is calculated by using (11) and is adopted as the new time-resolution of prediction model to perform the USTWPP within the prediction target time interval. As a result, the predicted values with the time-resolution $T_{u s}^{\prime}$ are obtained.

Step 3: Update the prediction target time interval along the timeline and go back to Step 2.

The proposed adaptive time-resolution method not only improves the prediction accuracy, but also ensures the prediction efficiency due to the following two features of the method. 
1) By mining the regularities of the relationship between $\rho_{\max H 0} \%$ and $k_{u s}$ first, and then adjusting the time-resolution in real time based on the mining results, the method can reduce the time cost of the adjustment.

2) By the interval grouping of $\rho_{\max H 0} \%$ and $k_{u s}$, the method can avoid the frequent adjustment of time-resolution with the real-time change of $\rho_{\max H 0} \%$, and consequently can reduce the storage space cost and time cost.

\section{Case Studies}

To verify the effectiveness of the proposed method, case studies with actual wind power data are carried out in this section. BPNN, one of the most widely used prediction models, is adopted for USTWPP in Matlab 2016a.

\subsection{Data and parameter settings}

The data in the case are selected from 28 days' wind power of a wind farm in northern China from May 10 to June 6, 2006, as shown in Fig. 4. The total capacity of the wind farm is $49.3 \mathrm{MW}$. The wind power data from May 10 to June 5 are taken as the training samples of USTWPP model, and the ultra-short-term wind power on June 6 is predicted on a rolling basis with adaptive time-solution according to the procedure in sub-section 3.3.

To demonstrate the prediction effects of USTWPP with adaptive time-solution, the prediction results in different scenarios on June 6 are analysed and compared with those with fixed time-resolution. Here the reference fixed time-resolution of prediction model $T_{\text {us } 0}$ is $15 \mathrm{~min}$, the number of time steps of the reference data used for analysing and predicting the fluctuation characteristics of HPE is $4(N=4)$ and the number of groups of $\rho_{\max H 0} \%$ and $k_{u s}$ is $6(M=6)$.

\subsection{Adjustment rules of time-resolution}

When the maximum hidden error rate $\delta_{\max H}$ in USTWPP with the reference time-resolution of 15 min is greater than the threshold $\delta_{\text {usth }}\left(\delta_{\max H}>\delta_{\text {usth }}\right)$, the adjustment rules of time-resolution after regularities mining and interval grouping optimization of $\rho_{\max H 0} \%$ and $k_{u s}$ are obtained according to sub-section 3.2, as shown in Table 3. 


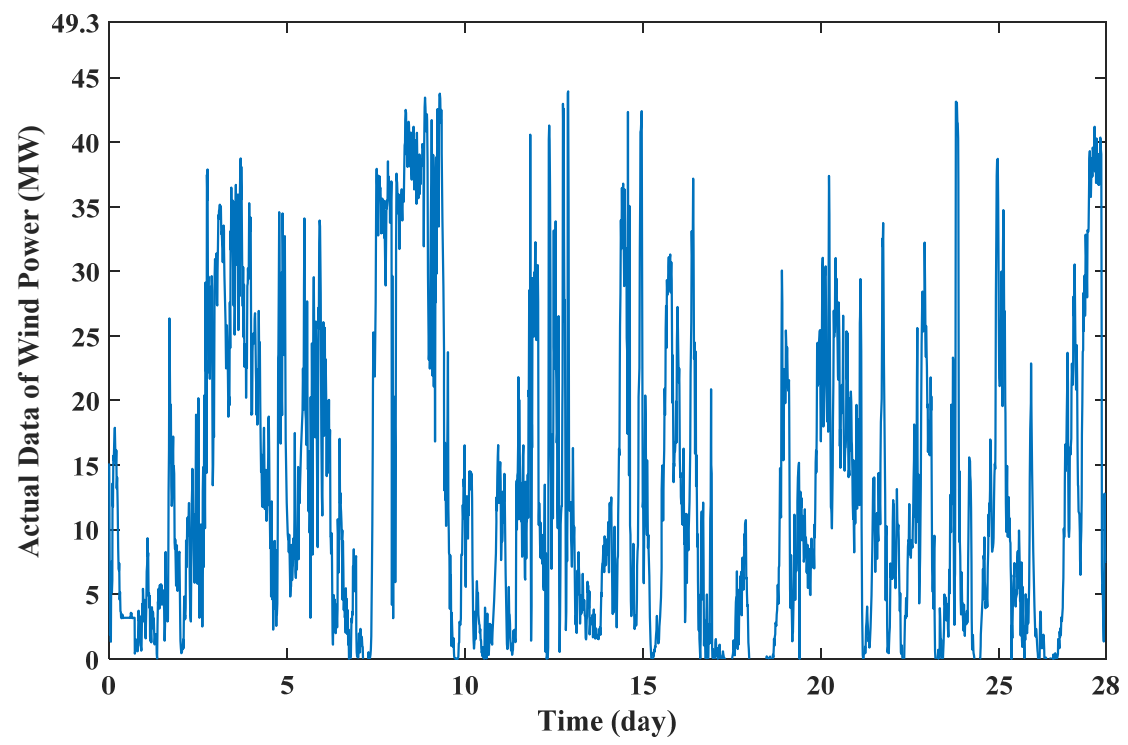

Fig. 428 days' actual data of wind power

Table 3 Adjustment rules of $\rho_{\max H 0} \%$ and $k_{u s}$

\begin{tabular}{cc}
\hline Range of $\rho_{\max H 0} \%$ & $k_{\text {us }}$ \\
\hline$\rho_{\max H 0} \% \in[0,0.0125]$ & $4 / 5$ \\
$\rho_{\max H 0} \% \in(0.0125,0.015]$ & $2 / 3$ \\
$\rho_{\max H 0} \% \in(0.015,0.018]$ & $8 / 15$ \\
$\rho_{\max H 0} \% \in(0.018,0.03]$ & $1 / 3$ \\
$\rho_{\max H 0} \% \in(0.03,0.05]$ & $1 / 5$ \\
$\rho_{\max H 0} \% \in(0.05,+\infty)$ & $1 / 15$ \\
\hline
\end{tabular}

\subsection{Prediction with adaptive time-resolution and comparison with fixed time-resolution}

During the rolling USTWPP on June 6, time-resolution is adaptively adjusted in real time based on the fluctuation characteristics of prediction error according to sub-section 3.3. The prediction results with adaptive time-resolution are compared with those with fixed time-resolution in the following three different scenarios, which demonstrates the adaptability and effectiveness of the proposed method.

Scenario 1: Predicting the wind power within the time interval from 12:15 to $16: 15$

In this scenario, the time $t_{0}$ is 12:15. The fluctuation characteristics of HPE of USTWPP with the reference time-resolution within $N$ time steps before time $t_{0}$ (from 11:15 to 12:15) are analysed. The maximum hidden error rate $\delta_{\max H}$ is obtained, which is less than the threshold $\delta_{\text {usth }}$. It is obvious that there is no wind power fluctuation with a large magnitude within the four time steps. Furthermore, according to the great temporal correlation in ultra-short-term wind power and the corresponding HPE se- 
1 ries, it can be predicted that the real-time wind power fluctuations will be accurately tracked by the prediction model with the reference time-resolution within the time interval from 12:15 to 16:15. Therefore, the reference time-resolution of $15 \mathrm{~min}$ is adopted in the prediction model to predict the wind power within the time interval from 12:15 to 16:15, as shown in Fig. 5.

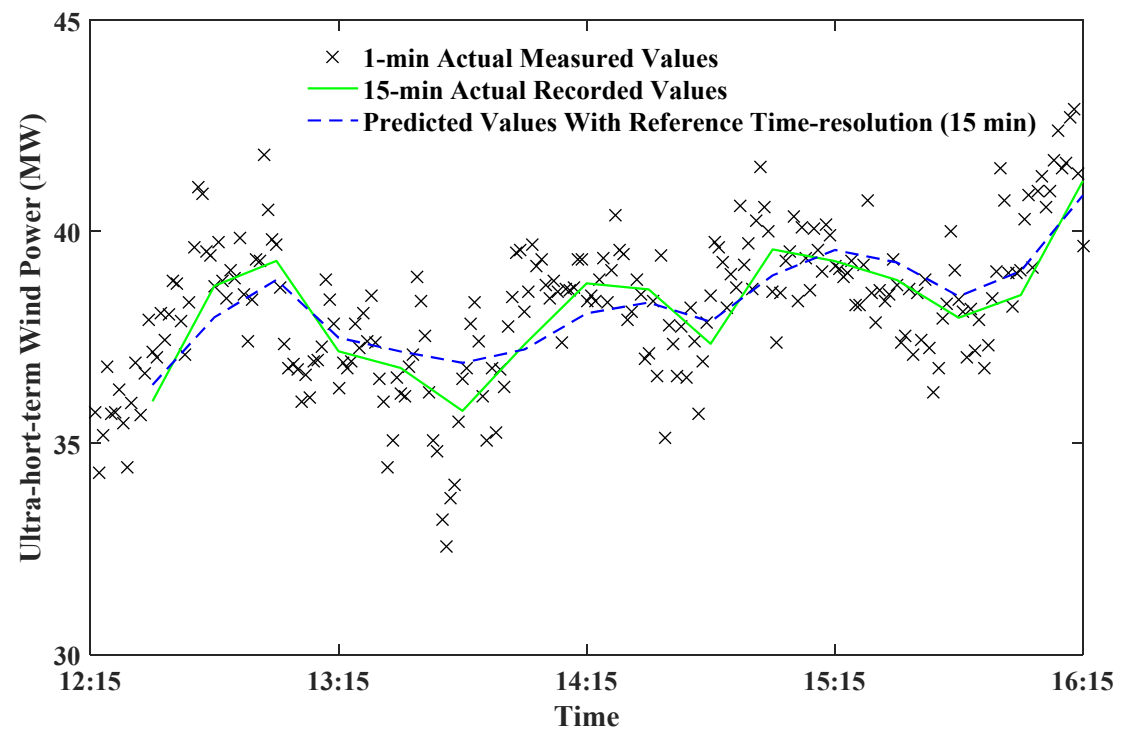

As can be seen from Fig. 5, the wind power within the time interval from 12:15 to 16:15 changes with no violent fluctuations. The prediction model with the reference time-resolution of 15 min accurately 9 predicts the wind power within the time interval.

The relative average prediction error $e_{\text {rel }}$ and relative HPE $e_{\text {Hrel }}$ within the time interval from 12:15 to 16:15 are obtained according to (2) and (4), as shown in Fig. 6.

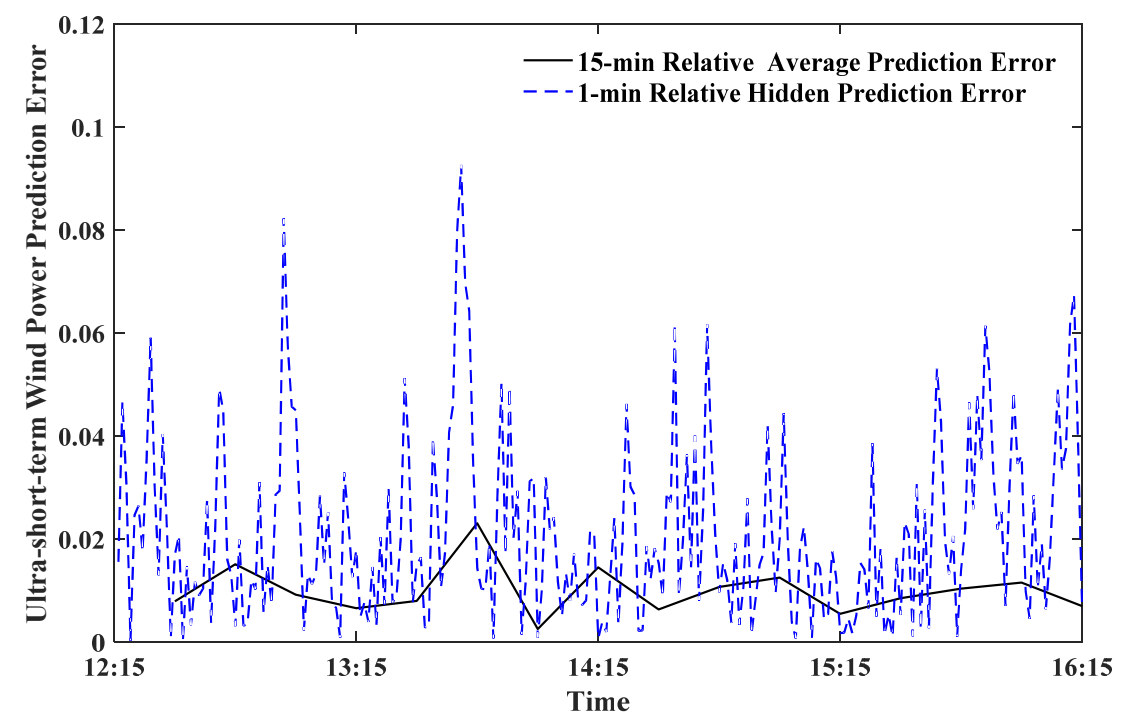

Fig. 6 Prediction error of USTWPP from 12:15 to 16:15 
It can be seen that the maximum hidden error rate of USTWPP with the reference time-resolution of 15 min within the time interval $\delta_{\max H}$ is 0.095 , less than the threshold $\delta_{\text {usth }}(0.095<0.15)$, which meets the performance requirements of prediction. Therefore, it demonstrates the validity of the proposed fluctuation characteristic analysis method of the prediction error in the scenarios without violent wind power fluctuations.

Scenario 2: Predicting the wind power within the time interval from 17:45 to 21:45

Similarly, the maximum hidden error rate $\delta_{\max H}$ and the maximum average monotonic fluctuation rate $\rho_{\max H_{0}} \%$ of HPE of USTWPP with reference time-resolution are calculated within the four time steps from 16:45 to $17: 45$. $\delta_{\max H}$ is 0.168 , greater than the threshold $\delta_{\text {usth }}(0.168>0.15)$, which indicates there are wind power fluctuations with large magnitudes within the four time steps. It can be prejudged that there will be large HPE if wind power is predicted with the reference time-resolution of 15 min within the time interval from 17:45 to $21: 45$. Therefore, it is necessary to adjust the time-resolution within the time interval. Since $\rho_{\max H 0} \%$ is $0.0176,8 / 15$ is selected as the adaptive adjustment coefficient of time-resolution $k_{u s}$ according to Table 3 and consequently the time-resolution is adjusted to 8 $\min \left(T_{u s}^{\prime}=8 \min \right)$.

The results of predicted values with adaptive time-resolution $(8 \mathrm{~min})$ and reference time-resolution (15 min) and actual values (1-min actual measured values and 15-min actual recorded values) within the time interval from 17:45 to 21:45 are shown in Fig. 7.

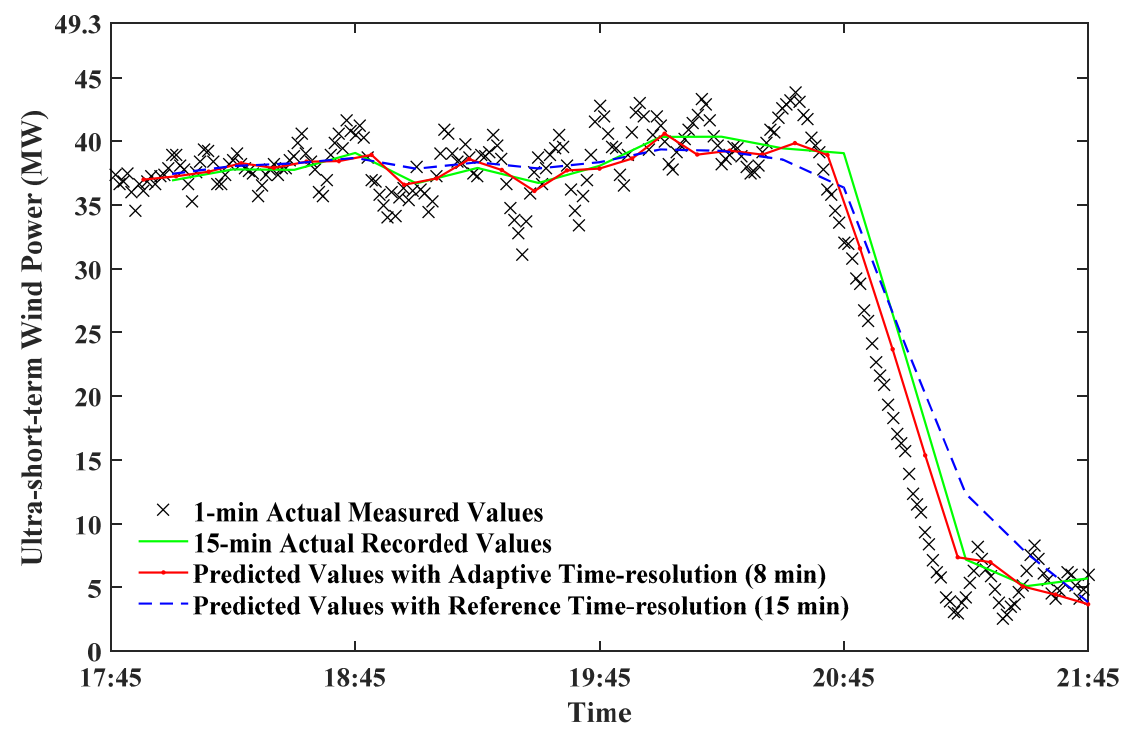

Fig. 7 Actual values and predicted values of wind power from 17:45 to 21:45 
It can be seen from Fig. 7 that the prediction model with adaptive time-resolution tracks the real-time fluctuations of wind power better than the prediction model with reference time-resolution does. Especially when wind power has violent fluctuations (20:30 - 21:30), the tracking effect is greatly improved.

The relative average prediction error $e_{\text {rel }}$ and relative HPE $e_{\text {Hrel }}$ within the time interval from 17:45 to 21:45 are obtained according to (2) and (4), as shown in Fig. 8.

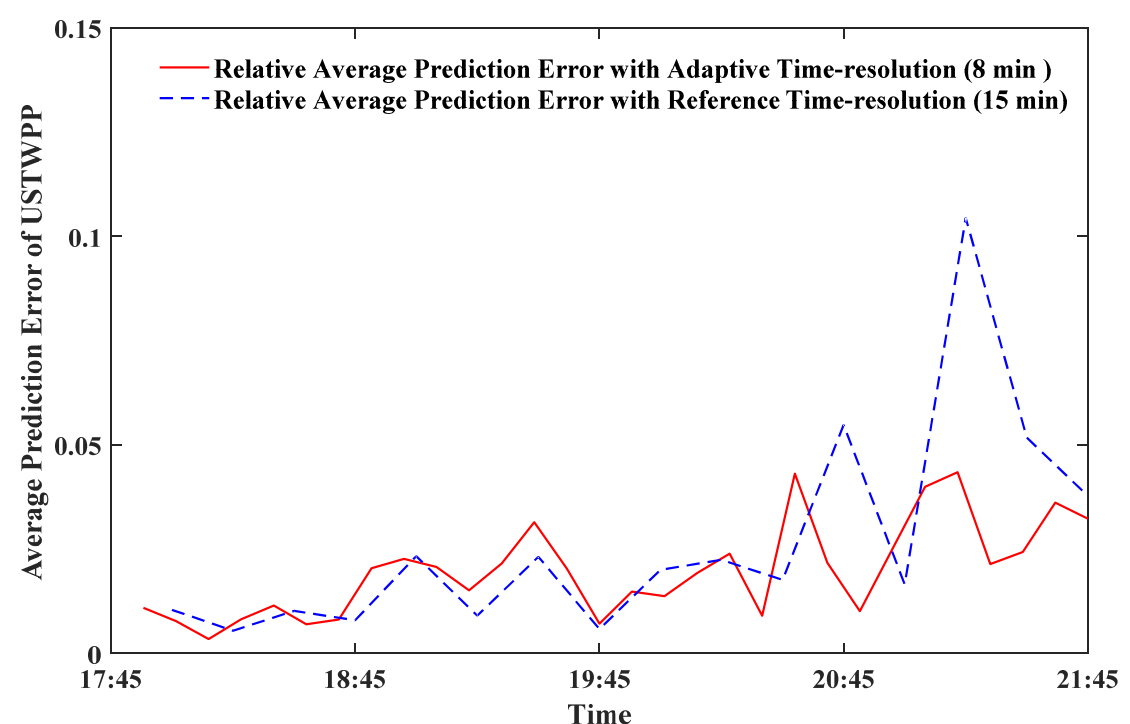

(a) Relative average prediction error from 17:45 to 21:45

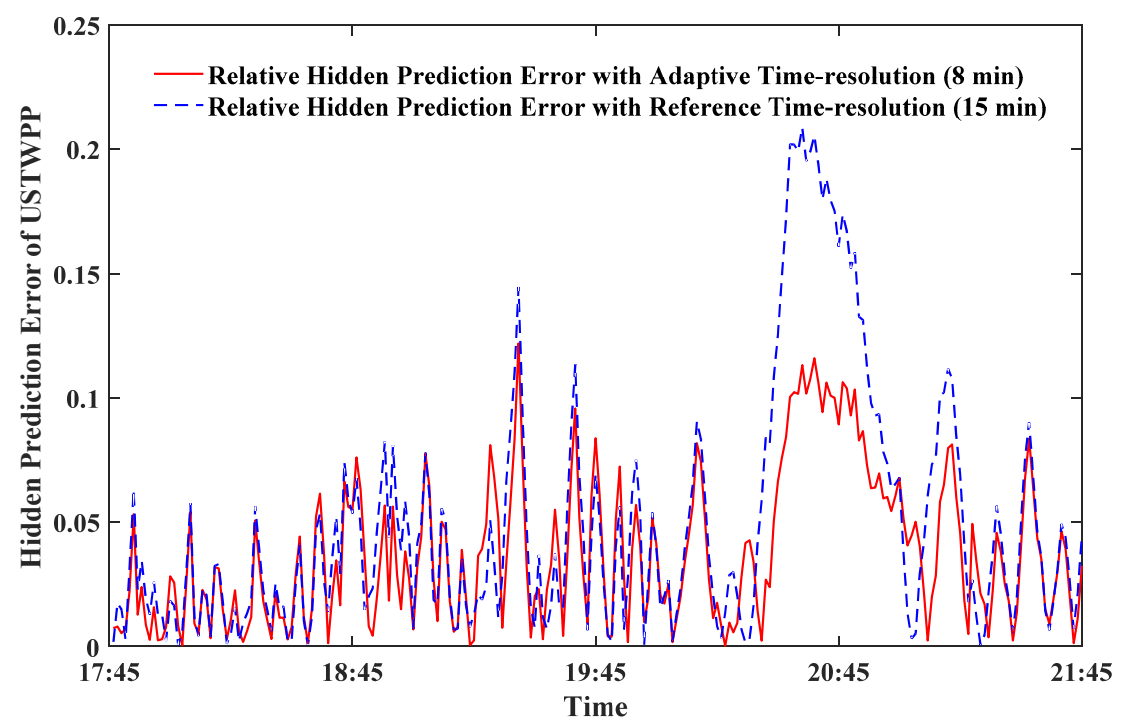

(b) Relative HPE from 17:45 to 21:45

Fig. 8 Prediction error of USTWPP with adaptive time-resolution and reference time-resolution from $17: 45$ to $21: 45$

By comparing Figs. 8(a) and 8(b), it can be observed that the relative HPE $e_{\text {Hrel }}$ can more accurately reflect the real-time dynamic fluctuations of prediction error than relative average prediction error $e_{\text {rel }}$. Moreover, there are large prediction errors within the time interval from 20:30 to 21:30 when wind power 
1 is predicted with reference time-resolution. The maximum hidden error rate $\delta_{\max H}$ with reference time-resolution within the time interval is 0.21 , which can threaten the security and stability of power systems. By applying adaptive time-resolution, $\delta_{\max H}$ within the time interval is reduced to 0.12 .

According to the prediction error evaluation indicators in Section 2, the comparison of relative average prediction error $e_{r e l}$ of USTWPP between adaptive time-resolution and reference time-resolution within the time interval from 17:45 to 21:45 is obtained, as shown in Table 4. The comparison of relative HPE $e_{\text {Hrel }}$ of USTWPP between adaptive time-resolution and reference time-resolution is shown in Table 5. An improvement degree indicator for each prediction error evaluation indicator is established to measure the effect of USTWPP with adaptive time-resolution as compared with that with fixed time-resolution. The improvement degree is calculated by dividing the difference between the evaluation indicators of USTWPP with adaptive time-resolution and reference time-resolution by the evolution indicator with reference time-resolution. The improvement degree indicators of relative average prediction error $e_{\text {rel }}$ and relative HPE $e_{H r e l}$ are listed in the fourth columns of Tables 4 and 5 respectively.

Table 4 Comparison of $e_{\text {rel }}$ between adaptive time-resolution and the reference time-resolution from

\begin{tabular}{cccc}
\hline Evaluation indicator & $\begin{array}{c}\text { USTWPP with adaptive } \\
\text { time-resolution }\end{array}$ & $\begin{array}{c}\text { USTWPP with reference } \\
\text { time-resolution }\end{array}$ & Improvement degree \\
\hline$\delta_{\max }$ & $\mathbf{0 . 0 4 3 5}$ & $\mathbf{0 . 1 0 4 4}$ & $\mathbf{5 8 . 3 3 \%}$ \\
$M A E$ & 0.0198 & 0.0263 & $24.71 \%$ \\
$R M S E$ & 0.0227 & 0.0362 & $37.29 \%$ \\
$A$ & 0.9773 & 0.9638 & $1.4 \%$ \\
\hline
\end{tabular}

Table 5 Comparison of $e_{\text {Hrel }}$ between adaptive time-resolution and the reference time-resolutions from $17: 45$ to $21: 45$

\begin{tabular}{cccc}
\hline Evaluation indicator & $\begin{array}{c}\text { USTWPP with adaptive } \\
\text { time-resolution }\end{array}$ & $\begin{array}{c}\text { USTWPP with reference } \\
\text { time-resolution }\end{array}$ & Improvement degree \\
\hline$\delta_{\max H}$ & $\mathbf{0 . 1 2 2 0}$ & $\mathbf{0 . 2 0 8 8}$ & $\mathbf{4 1 . 5 7 \%}$ \\
$M A E_{H}$ & 0.0379 & 0.0499 & $24.05 \%$ \\
$R M S E_{H}$ & 0.0482 & 0.0701 & $31.24 \%$ \\
$A_{H}$ & 0.9518 & 0.9299 & $2.36 \%$ \\
\hline
\end{tabular}

From the improvement degree indicators in Tables 4 and 5, all evaluation indicators of prediction error of USTWPP with adaptive time-resolution are improved as compared with USTWPP with reference time-resolution within the time interval. Especially, the maxi-mum error rate $\delta_{\max }$ and the maximum hidden error rate $\delta_{\max H}$ of USTWPP with adaptive time-resolution are $58.33 \%$ and $41.57 \%$, smaller than those of USTWPP with reference time-resolution, respectively. Furthermore, it can be seen that the $e_{\text {rel }}$ and $e_{\text {Hrel }}$ of USTWPP with adaptive time-resolution both meet the performance requirements of prediction. However, for the USTWPP with reference time-resolution, the maximum hidden error rate of rela- 
1 tive HPE $\delta_{\max H}$ is greater than the threshold $\delta_{\text {usth }}(0.2088>0.15)$ although $e_{\text {rel }}$ meets the performance requirements of prediction.

Therefore, it can be concluded that HPE can better evaluate the prediction performance and the prediction model with adaptive time-resolution has higher prediction accuracy within this time interval.

Scenario 3: Predicting the wind power within the time interval from 20:00 to 24:00

In this scenario, the maximum hidden error rate $\delta_{\max H}$ of HPE of USTWPP with reference time-resolution within $N$ time steps before prediction target time interval is 0.153 , greater than the threshold $\delta_{\text {usth }}(0.153>0.15)$ and the maximum average monotonic fluctuation rate $\rho_{\max H 0} \%$ is 0.028 . Therefore, for USTWPP within the time interval from 20:00 to 24:00, 1/3 is selected as the adaptive adjustment coefficient of time-resolution $k_{u s}$ and then the time-resolution is adjusted to $5 \mathrm{~min}\left(T_{u s}^{\prime}=5 \mathrm{~min}\right.$ ) .

The results of predicted values with adaptive time-resolution $(5 \mathrm{~min})$ and reference time-resolution (15 min) and actual values (1-min actual measured values and 15-min actual recorded values) within the time interval from 20:00 to 24:00 are shown in Fig. 9.

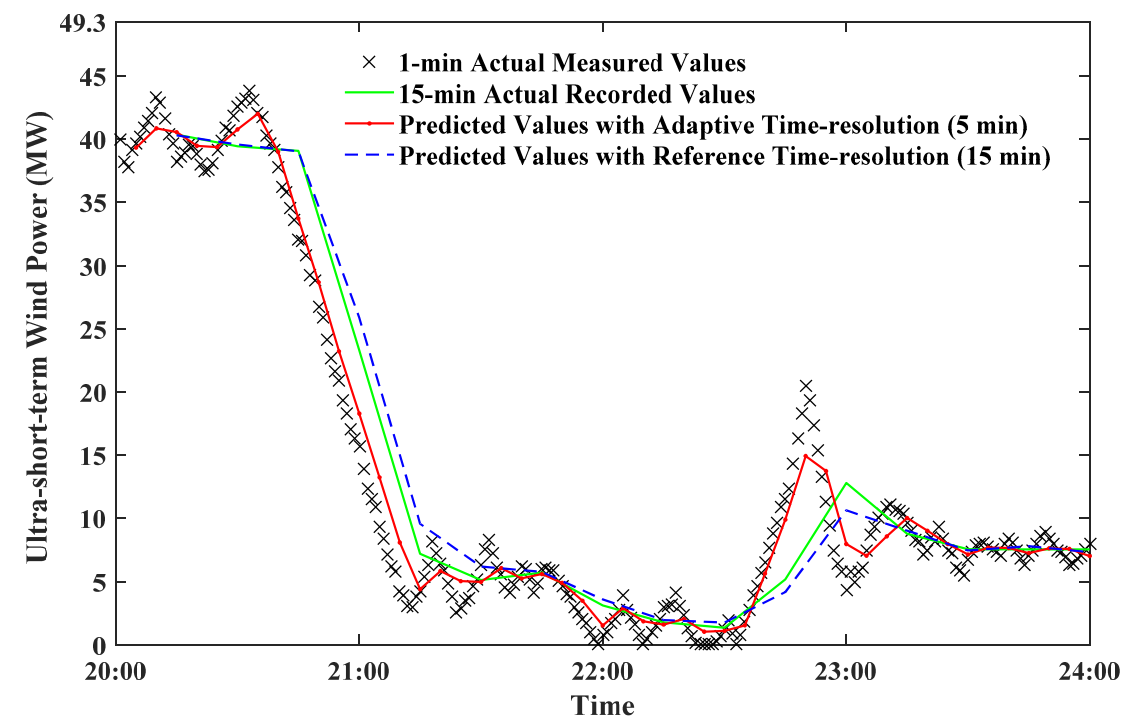

Fig. 9 Actual values and predicted values of wind power from 20:00 to 24:00

Within this time interval, the prediction model with adaptive time-resolution tracks the real-time fluctuations of wind power better than the prediction model with reference time-resolution does. Especially when wind power has violent fluctuations (20:45 - 21:30 and 22:45 - 23:15), the tracking effect is greatly improved.

The relative average prediction error $e_{r e l}$ and relative HPE $e_{H r e l}$ within the prediction target time interval are shown in Fig. 10. 


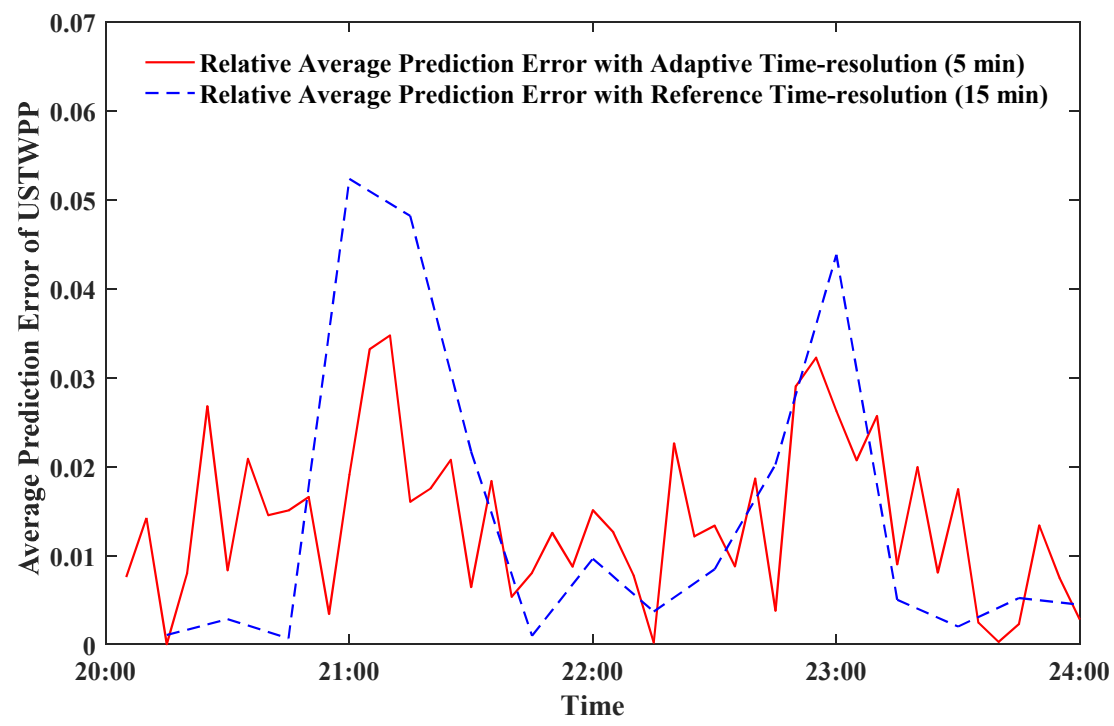

(a) Relative average prediction error from 20:00 to 24:00

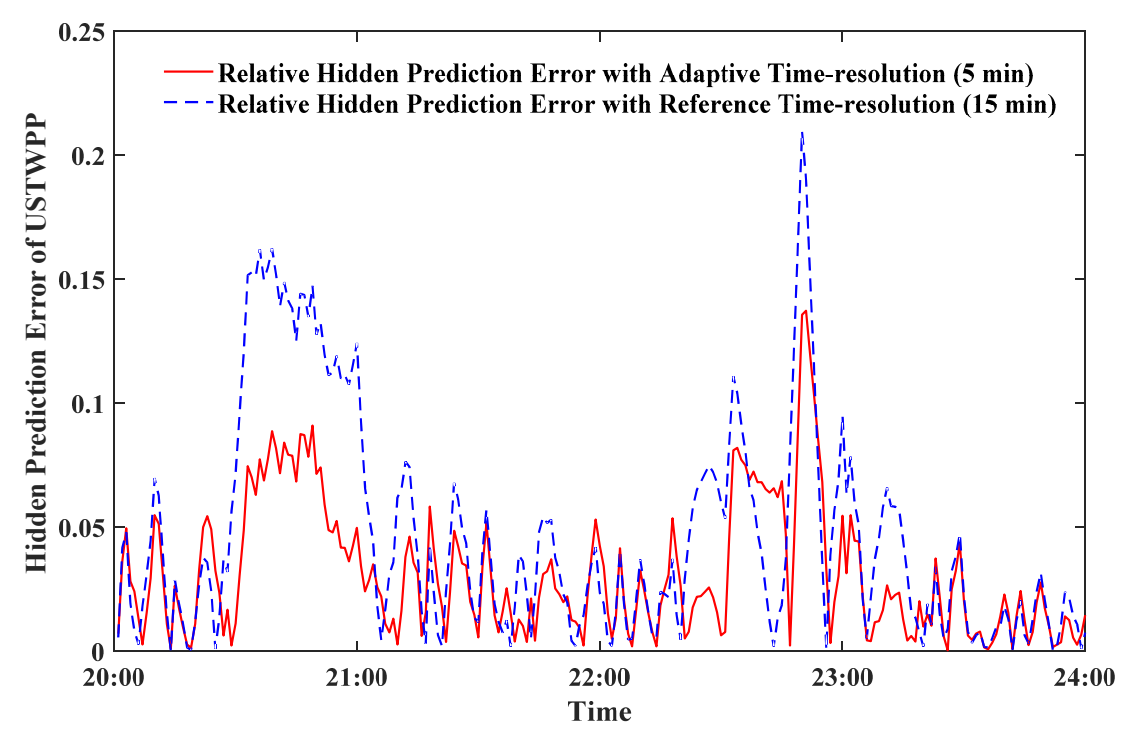

(b) Relative HPE from 20:00 to 24:00

Fig. 10 Prediction error of USTWPP with adaptive time-resolution and the reference time-resolution from 20:00 to $24: 00$

It can be observed that there are large prediction errors within the time intervals from 20:45 to 21:30 and from 22:45 to 23:15 when wind power is predicted with reference time-resolution. The maximum hidden error rates $\delta_{\max H}$ within the two time intervals are 0.16 and 0.21 , respectively, which are beyond the accuracy requirements. However, $\delta_{\max H}$ within the two time intervals are reduced to 0.08 and 0.14 in the USTWPP with adaptive time-resolution, respectively, both meeting the accuracy requirements.

The comparison of prediction error of USTWPP between adaptive time-resolution and the reference time-resolution within the time interval are given in Tables 6 and 7. 
Table 6 Comparison of $e_{\text {rel }}$ between adaptive time-resolution and the reference time-resolution from 20:00 to $24: 00$

\begin{tabular}{cccc}
\hline Evaluation indicator & $\begin{array}{c}\text { USTWPP with adaptive } \\
\text { time-resolution }\end{array}$ & $\begin{array}{c}\text { USTWPP with reference } \\
\text { time-resolution }\end{array}$ & Improvement degree \\
\hline$\delta_{\max }$ & 0.0347 & 0.0523 & $33.65 \%$ \\
$M A E$ & 0.0139 & 0.0144 & $3.47 \%$ \\
$R M S E$ & 0.0165 & 0.0225 & $26.67 \%$ \\
$A$ & 0.9835 & 0.9775 & $0.61 \%$ \\
\hline
\end{tabular}

Table 7 Comparison of $e_{\text {Hrel }}$ between adaptive time-resolution and the reference time-resolutions from 20:00 to $24: 00$

\begin{tabular}{cccc}
\hline Evaluation indicator & $\begin{array}{c}\text { USTWPP with adaptive } \\
\text { time-resolution }\end{array}$ & $\begin{array}{c}\text { USTWPP with reference } \\
\text { time-resolution }\end{array}$ & Improvement degree \\
\hline$\delta_{\max H}$ & $\mathbf{0 . 1 3 7 3}$ & $\mathbf{0 . 2 0 9 7}$ & $\mathbf{3 4 . 5 3 \%}$ \\
$M A E_{H}$ & 0.0314 & 0.0473 & $33.62 \%$ \\
$R M S E_{H}$ & 0.0412 & 0.0654 & $37.00 \%$ \\
$A_{H}$ & 0.9588 & 0.9346 & $2.59 \%$ \\
\hline
\end{tabular}

It can be seen from the above comparisons in the Tables 6 and 7 that the prediction accuracy of USTWPP with adaptive time-resolution are improved in contrast to USTWPP with reference time-resolution within the time interval. The $e_{r e l}$ and $e_{\text {Hrel }}$ of USTWPP with adaptive time-resolution both meet the performance requirements of prediction. However, for the USTWPP with reference time-resolution, the maximum hidden error rate of relative HPE $\delta_{\max H}$ is greater than the threshold $\delta_{\text {usth }}$ $(0.2097>0.15)$ although $e_{\text {rel }}$ meets the performance requirements of prediction.

From the above different scenarios, the time-resolution of USTWPP is adaptively adjusted according to the fluctuation characteristics of prediction error based on the proposed method. The prediction accuracy of USTWPP with adaptive time-resolution is higher than that of USTWPP with reference fixed time-resolution, which avoids the phenomenon that the threshold requirements of maximum hidden error rate cannot be met when the wind power has violent fluctuations.

\subsection{Prediction with adaptive time-resolution and comparison with other prediction models}

In order to further evaluate the performance of proposed method, the prediction results of model with adaptive time-resolution are compared to those generated by benchmark wind power prediction models under Scenario 3, such as BPNN with adaptive time-scale, ARIMA [36], ELM [18] and SVM [16].

The predicted values of different models are shown in Fig. 11. It can be seen that prediction values with adaptive time-resolution are closest to the actual values, which indicates that the prediction model 
1 with adaptive time-resolution can reflect the real-time fluctuation trend of wind power better than other prediction models.

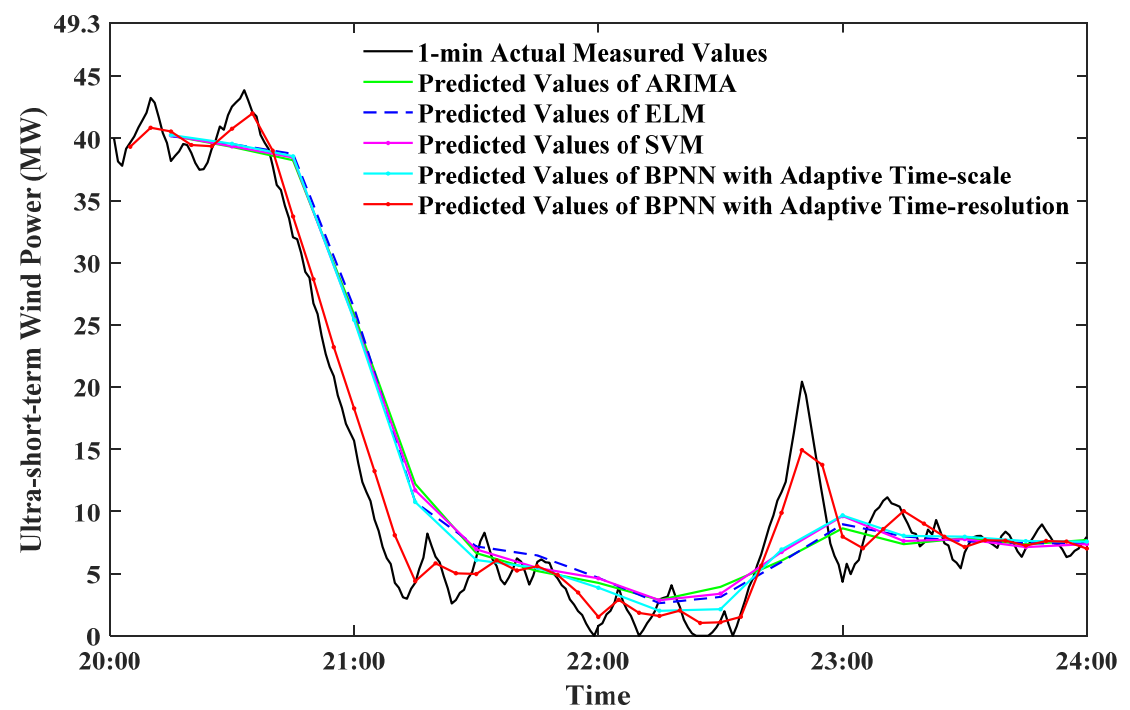

Fig. 11 Wind power actual values and predicted values of different models

The relative average prediction error $e_{r e l}$ and relative HPE $e_{\text {Hrel }}$ are depicted in Fig. 12. The average prediction error of proposed model with adaptive time-resolution is much smaller than that of other models, and the corresponding HPE does not exceed 15\% even when wind power has extremely violent 8 fluctuations, which overcomes the problem that the threshold requirements of prediction accuracy cannot 9 be met during the prediction of other models.

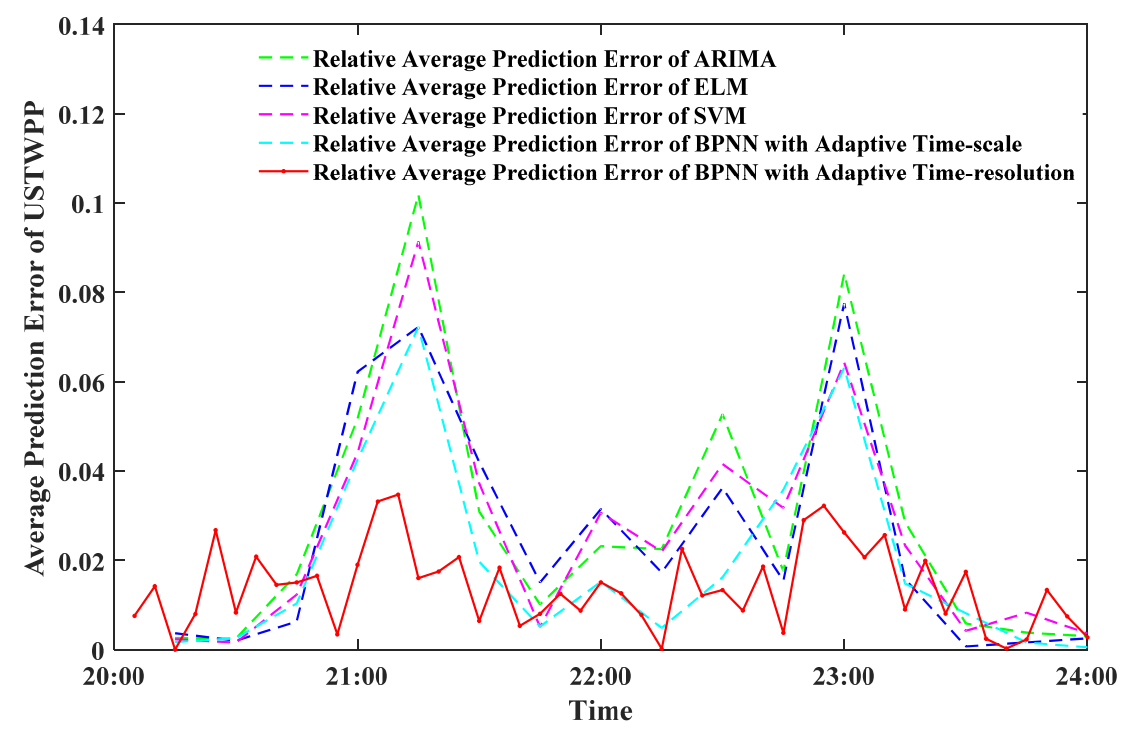

(a) Relative average prediction error of different models 


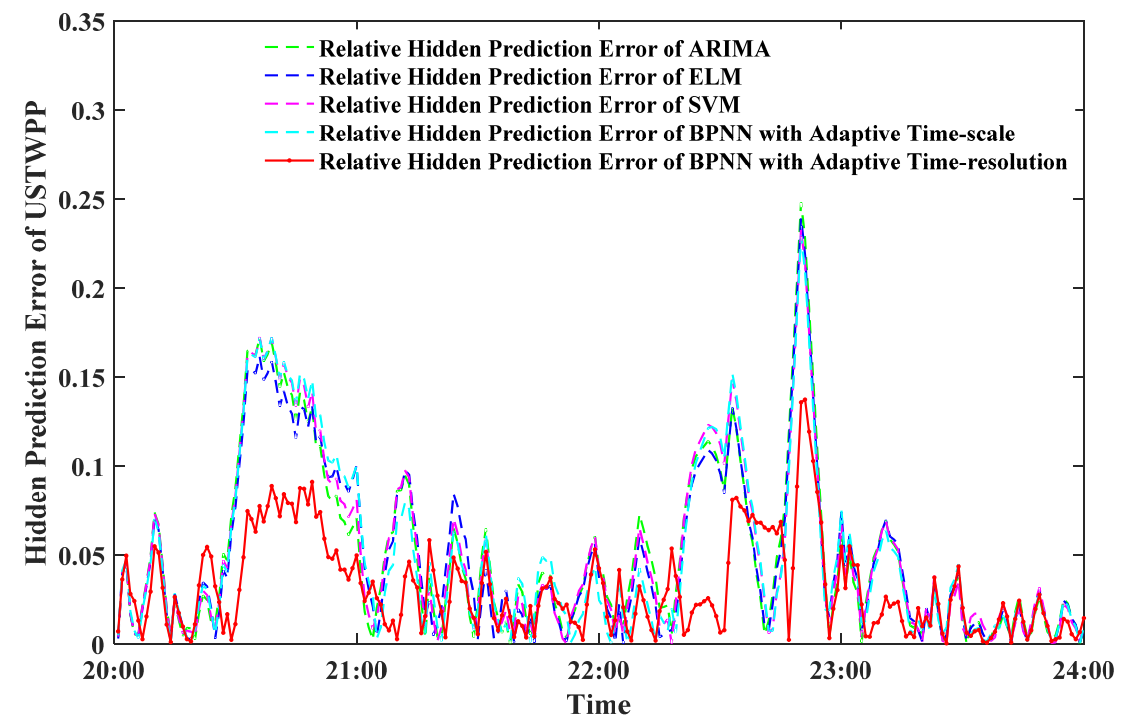

(b) Relative HPE of different models

Fig. 12 Prediction error of different models

The comparison results of prediction error evaluation indicators of different models are presented in Table 8 . The values in the second to fifth columns and the fifth to ninth columns represent the indicators of $e_{r e l}$ and $e_{\text {Hrel }}$, respectively.

Table 8 Comparison of prediction error evaluation indicators of different models

\begin{tabular}{ccccccccc}
\hline \multirow{2}{*}{ Prediction model } & \multicolumn{8}{c}{ Evaluation indicator } \\
\cline { 2 - 9 } & $\delta_{\max }$ & $M A E$ & $R M S E$ & $A$ & $\delta_{\max H}$ & $M A E_{H}$ & $R M S E_{H}$ & $A_{H}$ \\
\hline ARIMA & 0.1019 & 0.0287 & 0.0407 & 0.9593 & $\mathbf{0 . 2 4 7 8}$ & 0.0506 & 0.0701 & 0.9299 \\
ELM & 0.0776 & 0.0252 & 0.0356 & 0.9644 & $\mathbf{0 . 2 3 9 2}$ & 0.0497 & 0.0684 & 0.9316 \\
SVM & 0.0914 & 0.0265 & 0.0361 & 0.9639 & $\mathbf{0 . 2 3 2 8}$ & 0.0512 & 0.0710 & 0.9290 \\
BPNN with adaptive time-scale & 0.0723 & 0.0197 & 0.0292 & 0.9708 & $\mathbf{0 . 2 2 9 2}$ & 0.0502 & 0.0706 & 09294 \\
BPNN with adaptive time-resolution & 0.0347 & 0.0139 & 0.0165 & 0.9835 & $\mathbf{0 . 1 3 7 3}$ & 0.0314 & 0.0412 & 0.9588 \\
\hline
\end{tabular}

It is clear that the prediction error evaluation indicators of prediction model with adaptive time-resolution are smaller than those of other models. Therefore, the proposed model outperforms the benchmarks. In terms of the maximum hidden error rate $\delta_{\max H}$, the prediction model with adaptive time-resolution has a $40.10 \%-44.59 \%$ improvement compared to the benchmarks. The prediction results of the proposed model meet the accuracy requirements that $\delta_{\max H}$ should not exceed $15 \%$, while other models do not. There are also $52.01 \%-65.95 \%, 29.44 \%-51.56 \%, 43.49 \%-59.46 \%, 1.29 \%-2.52 \%$, $36.82 \%-38.67 \%, 41.97 \%-39.77 \%$ and $2.84 \%-3.11 \%$ improvements on $\delta_{\max }, M A E, R M S E, A$, $M A E_{H}, R M S E_{H}$ and $A_{H}$, respectively.

The outstanding performance of the proposed method owes that it can adaptively adjust the prediction time-resolution according to the fluctuation characteristics of HPE to make the prediction model with 
1 adaptive time-resolution accurately track the real-time fluctuations of wind power. Therefore, the risks that the threshold requirements of $\delta_{\max H}$ are not met can be avoided even when wind power fluctuates 3 violently.

\section{Conclusions}

In this paper, an adaptive time-resolution method for USTWPP is proposed to improve the prediction accuracy, especially under the situations of extremely violent fluctuations of wind power. It has been demonstrated that the HPE can better reveal the real-time fluctuation characteristics of wind power within the time step, and the prediction time-resolution can be adaptively adjusted with the proposed method. Case studies and comparison results indicate that the prediction model with adaptive time-resolutions has smaller prediction error in contrast to the existing models, and the maximum hidden error rate of HPE can be reduced by $44.59 \%$ when the wind power has extremely violent fluctuations. It should be noted that the proposed adaptive time-resolution method can also be applied to other WPP models to predict wind power. Furthermore, it is easily extended and used in the short-term WPP and the other prediction application fields with violent fluctuation characteristics, such as solar power and load.

\section{Acknowledgment}

This work was jointly supported by the National Natural Science Foundation of China (51877072, 51307148).

\section{References}

[1] Badal FR, Das P, Sarker SK, et al. A survey on control issues in renewable energy integration and microgrid. Protection and Control of Modern Power Systems 2019; 4(1): 87-113.

[2] Liang XD. Emerging power quality challenges due to integration of renewable energy sources. IEEE Transactions on Industry Applications 2017; 53(2): 855-866.

[3] Wang HZ, Lei ZX, Zhang X, et al. A review of deep learning for renewable energy forecasting. Energy Conversion and Management 2019;198.

[4] Moghaddam, SZ. Generation and transmission expansion planning with high penetration of wind farms considering spatial distribution of wind speed. International Journal of Electrical Power \& Energy Systems 2019; 106: $232-241$.

[5] Zheng DH, Eseye AT, Zhang JH, et al. Short-term wind power forecasting using a double-stage hierarchical ANFIS approach for energy management in microgrids. Protection and Control of Modern Power Systems 2017; 2(2): 136-145. 
[6] Yan J, Liu YQ, Han S, et al. Reviews on uncertainty analysis of wind power forecasting. Renewable and Sustainable Energy Reviews 2015; 52: 1322-1330.

[7] Xue YS, Yu C, Li K, et al. Adaptive ultra-short-term wind power prediction based on risk assessment. CSEE Journal of Power and Energy Systems 2016; 2(3): 59-64.

[8] Hong DY, Ji TY, Li MS, et al. Ultra-short-term forecast of wind speed and wind power based on morphological high frequency filter and double similarity search algorithm. International Journal of Electrical Power \& Energy Systems 2019; 104: 868-879.

[9] Fang RM, Wang YD, Shang RY, et al. The ultra-short-term power prediction of wind farm considering operational condition of wind turbines. International Journal of Hydrogen Energy 2016; 41(35): 15733-15739.

[10] Ma L, Luan SY, Jiang CW, et al. A review on the forecasting of wind speed and generated power. Renewable and Sustainable Energy Reviews 2009; 13(4): 915-920.

[11] Khalid M, Savkin AV. A method for short-term wind power prediction with multiple observation points. IEEE Transactions on Power Systems 2012; 27(2): 579-586.

[12] Zhao YN, Ye L, Li Z, et al. A novel bidirectional mechanism based on time series model for wind power forecasting. Applied Energy 2016; 177: 793-803.

[13] Wang SX, Zhang N, Wu L, et al. Wind speed forecasting based on the hybrid ensemble empirical mode decomposition and GA-BP neural network method. Renewable Energy 2016; 94: 629-636.

[14] Poncela M, Poncela P, Perán JR. Automatic tuning of Kalman filters by maximum likelihood methods for wind energy forecasting. Applied energy 2013; 108: 349-362.

[15] Shi J, Ding ZH, Lee WJ, et al. Hybrid forecasting model for very-short term wind power forecasting based on grey relational analysis and wind speed distribution features. IEEE Transactions on Smart Grid 2013; 5(1): 521-526.

[16] Kong XB, Liu XJ, Shi RF, et al. Wind speed prediction using reduced support vector machines with feature selection. Neurocomputing 2015; 169: 449-456.

[17] Chen P, Pedersen T, Bak-Jensen B, et al. ARIMA-based time series model of stochastic wind power generation. IEEE Transactions on Power Systems 2010; 25(2): 667-676.

[18] Wan C, Xu Z, Pinson P, et al. Probabilistic forecasting of wind power generation using extreme learning machine. IEEE Transactions on Power Systems 2014; 29(3): 1033-1044.

[19] Zhang R, Dong ZY, Xu Y, et al. Short-term load forecasting of Australian national electricity market by an ensemble model of extreme learning machine. IET Generation, Transmission \& Distribution 2013; 7(4): 391-397.

[20] Wan C, Xu Z, Pinson P, et al. Optimal prediction intervals of wind power generation. IEEE Transactions on Power Systems 2014; 29(3): 1166-1174.

[21] Wan C, Lin J, Wang JH, et al. Direct quantile regression for nonparametric probabilistic forecasting of wind power generation. IEEE Transactions on Power Systems 2017; 32(4): 2767-2778.

[22] Wang KJ, Qi XX, Liu HD, et al. Deep belief network based k-means cluster approach for short-term wind power forecasting. Energy 2018; 165: 840-852. 
[23] Hong WC. Application of seasonal SVR with chaotic immune algorithm in traffic flow forecasting. Neural Computing and Applications 2012; 12(3): 583-593.

[24] Zhang YG, Chen B, Zhao Y, et al. Wind speed prediction of IPSO-BP neural network based on Lorenz Disturbance. IEEE Access 2018; 6: 53168-53179.

[25] Alencar DB, Affonso CM, Oliveira RCL, et al. Hybrid approach combining SARIMA and neural networks for multi-step ahead wind speed forecasting in Brazil. IEEE Access 2018; 6: 55986-55994.

[26] Zhu S, Yuan XH, Xu ZY, et al. Gaussian mixture model coupled recurrent neural networks for wind speed interval forecast. Energy Conversion and Management 2019;198.

[27] Ju Y, Sun GY, Chen QH, et al. A model combining convolutional neural network and LightGBM algorithm for ultra-short-term wind power forecasting. IEEE Access 2019; 7: 28309-28318.

[28] Xiao CY, Wang NB, Ding K, et al. System power regulation scheme for Jiuquan wind power base. Proceedings of the CSEE 2010; 30(10): 1-7. (in Chinese).

[29] Wan Y. Analysis of wind power ramping behavior in ERCOT, http://digital-library.theiet.org/journals/author-guide; 2014 [accessed 27 November 2014].

[30] Bao ZJ, Zhou Q, Yang ZH, et al. A multi time-scale and multi energy-type coordinated microgrid scheduling solution-part i: model and methodology. IEEE Transactions on Power Systems 2015; 30(5): 2257-2266.

[31] Che L, Liu X, Zhu X, et al. Intra-interval security based dispatch for power systems with high wind penetration. IEEE Transactions on Power Systems 2019; 34(2): 1243-1255.

[32] Che L, Liu X, Li ZY. An Intra-interval security risk regarding regulation burden due to wind variation in high-wind-penetrated power systems. IEEE Transactions on Power Systems 2018; 33(3): 3213-3216.

[33] ICS 29.240. Function specification of wind power forecasting. State Grid Corporation of China 2015. (in Chinese).

[34] National energy administration of China. Interim measures for power prediction and prediction management of wind farm. Acta Energiae Solaris Sinica 2011(14): 6-7. (in Chinese).

[35] Zjavka L, Mišák S. Direct wind power forecasting using a polynomial decomposition of the general differential equation. IEEE Transactions on Sustainable Energy 2018; 9(4): 1529-1539.

[36] Yunus K, Thiringer T, Chen PY. ARIMA-based frequency-decomposed modelling of wind speed time series. IEEE Transactions on Power Systems 2016; 31(4): 2546-2556. 